\title{
Aiming for G7 Master Compliance through a Color Managed Workflow: Comparison of Compliance with Amplitude Modulated (AM) vs. Frequency Modulated (FM) Screening of Multicolor Digital Printing
}

\section{ABSTRACT}

The purpose of this research was to determine the influence of screening technologies (AM vs. FM) in the color reproduction aimed at the G7 master compliance. The quality of digital color printing is determined by these influential factors: screening method applied, type of printing process, ink (dry-toner or liquid-toner), printer resolution and the substrate (paper). For this research, only the color printing attributes such as the G7 colors hue and chroma, gray balance, and overall color deviations were analyzed to examine the significant differences that exist between the two screening technologies (AM vs. FM). These are the color attributes which are monitored and managed for quality accuracy during the printing. Printed colorimetry of each screening from the experiment was compared against G7 ColorSpace GRACOL 2013 (CGATS21-2-CRPC6) in CIE $L^{*} a^{*} b^{*}$ space using an IDEAlliance (Chromix/Hutch Color) Curve 4.2.4 application interface with an X-Rite spectrophotometer with an i1iO table. The measured data of each screening were run through this application (Curve 4.2.4). The data of each screening were analyzed by using the Verify Tool of the Curve 4.2.4 application to determine the pass/fail of G7 master compliance levels using G7 ColorSpace tolerances (G7 Grayscale, G7 Targeted, and G7 Colorspace). Analyzed data from the experiment revealed that the printed colorimetric values of each screening (G7 Grayscale, G7 Targeted, and G7 Colorspace) are in match (aligned) with the G7 master compliance levels (reference/target) colorimetric values (G7 Grayscale, G7 Targeted, and G7 Colorspace). Therefore, the press run was passed by the Curve 4 application for both screening technologies tested.

\author{
Haji Naik Dharavath (1) \\ Central Connecticut State \\ University, Department of \\ Computer Electronics \& Graphics \\ Technology, New Britain, USA \\ Corresponding author: \\ Haji Naik Dharavath \\ e-mail:dharavathh@ccsu.edu
}

First received: 15.12 .2020 .

Accepted: 25.2.2021.

\section{KEY WORDS}

G7, calibration, color, colorimetry, gamut, profiling, screening

\section{Introduction}

A continuous-tone color image is composed of a full spectrum of shades and color, from near white to dense black. In a traditional printing (offset, digital offset, gravure or flexography) workflow, the method by which continuous-tone photographic images are transformed to a printable image is called halftoning. In this method, varying percentages of the printed sheet are covered with halftone dots to represent the varying tones in the image. The ink (paste or liquid ink or dry toner) printed by each dot, of course, has the same density. At normal viewing distance, the dots of a printed image create an optical illusion of a continuous tone image. In contrast, a 
simple digital image could be a binary picture, $[h(x, y)]$, with each point being either completely black or completely white (Pnueli \& Bruckstein, 1996). A digital halftone is a pixel map, with bit depth, that gives the impression of an image containing a range of gray shades or continuous tones. An 8-bit grayscale image contains 256 different levels of gray from white to black.

A modern and up-to-date commercial printing workflow requires a Color Management System (CMS) to produce a quality color printing. A CMS enables the color producer (printer operator or the designer) to deliver accurate output colors regardless of device color capacities with the use of proper color management techniques (see Figure 1). Analyzing the color image by examining its quantitative attributes eliminates the subjective judgment of color quality evaluation of printed colors or colors in nature. Advancements in science and engineering, however, have allowed print and graphic professionals to apply scientific research methods across printing workflow. Applying these methods heightens the importance of proper print production workflow.

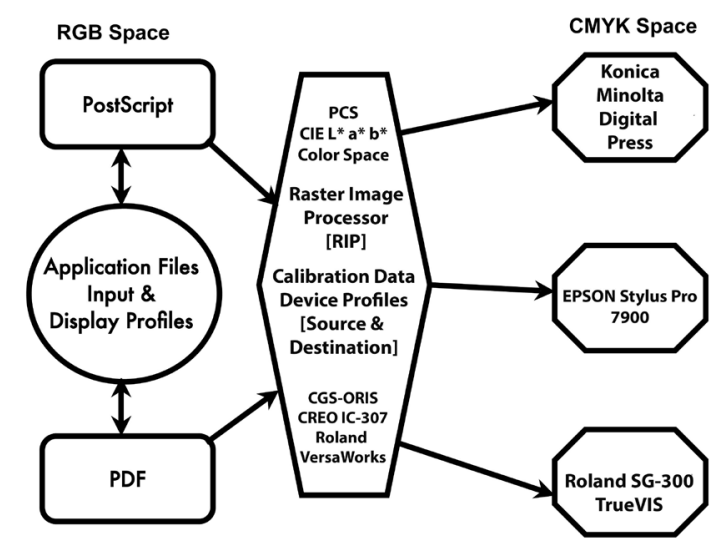

» Figure 1: Schematics of Color Management System (CMS)

\section{Rationale for literature review}

Workflow is represented through schematic illustrations of activities that reflect the systematic organization of analog and digital devices used during the print and image production process. A print ready e-file (.PDF or .JPEG or .PSD or PostScript, etc.) is likely to be manipulated and later printed by an array of output digital devices [computer-to-plate (CTP), digital printers and printing presses]. Given each family of devices tends to create and produce color differently, the challenge is to manage color consistency across the entire workflow. Digital color print reproduction involves physical/mechanical interaction among the imaging cylinder, dry/liquid toner, and the substrate (Novaković \& Avramović, 2012). The outcome of this interaction is the color print. Color can be viewed as a science where the optical aspects of color are quantitatively analyzable and measurable. The human eye, however, perceives color more subjectively, which poses a challenge at times for the printing and image reproduction industry.

G7 stands for grayscale (or gray) plus the seven primary and secondary colors known as the subtractive and additive: Cyan, Magenta, Yellow, Black (CMYK) and Red, Green, Blue (RGB). G7 is a method which specifies calibration procedures for printing visually acceptable colors with an emphasis on matching colorimetrically derived aim-points for the print reproduction processes to print with a common visual appearance. Today, this method (G7) is used in many applications of printing such as offset lithography, flexography, and digital (color laser or inkjet). It uses a pre-defined one-dimensional neutral print density curve (NPDC) to match neutral tonality/gray balance. $\mathrm{G} 7$ specifications are owned by International Digital Enterprise Alliance (International Digital Enterprise Alliance- IDEAlliance, 2014) and the colorimetric formulas of the G7 are defined in the American National Standards Institute and the Committee on Graphic Arts Technology Standards/Technical Report (ANSI/CGATS TR015). Published reports reveal there are three ways G7 master compliance can be achieved: a) output device NPDC to G7 NPDC [P2P251x target image], b) use of output device ICC profile, and c) the use of device link profile (DLP = source as GRACoL2013 ICC profile + the destination device ICC profile). G7 master compliance includes three levels in the $G 7$ master qualification: $G 7$ Grayscale, G7 Targeted, and G7 Colorspace. These levels demonstrate $\mathrm{G} 7$ master capabilities of a print facility.

\section{G7 Grayscale}

This is the fundamental level of G7 commonly seen in most color print reproduction. Regardless of printing process, if a digital printer or printing press reproduces the defined neutral tone ramp as a neutral gray, then all other colors in the reproduction are believed to be without colorcast. This is determined by printing a target specified on a stable printing system and then measuring the target using the correct ink/toner curves to bring the printing system into alignment with the $\mathrm{G} 7$ ideal neutral density curve. Aligning the various reproduction processes and obtaining the same neutral aim points is critical for consistent reproduction.

\section{G7 Targeted}

The secondary level of G7 is achieved when G7 grayscale is matched and the solid ink measurements for primary and secondary (CMY and RGB) are also within the G7 target specifications. This can be achieved through the absolute white point or using the substrate-relative conditions. However, G7 Targeted compliance is not limited to the reference print conditions in ISO 12647-2 or in ISO/ 
PAS 15339. The G7-calibrated dataset can be used as a G7 reference print condition. G7 Targeted achievement certifies that the facility not only conforms to G7 Grayscale, but it can also achieve a higher level of compliance.

\section{G7 Colorspace}

The highest level of $\mathrm{G} 7$ compliance and the most stringent is the G7 Colorspace. It includes all the requirements of the G7 Targeted level and, therefore, the G7 Grayscale level. This also includes the matching of an entire Reference Print Condition (RPC). This level of control demonstrates that the reproduction maintains an extremely tight tolerance throughout the complete color space. An entire TC1617x target is printed and compared against the specific color space with all 1617 patches held to within a tight tolerance. This assures the printing system will reproduce the entire color space, not just the primary and secondary colors of CMYK and RGB. The G7 Colorspace can also relate to either the absolute white point or the substrate-relative aim values.

Regardless what experts (published reports) say, there is no single/fixed screening method that would work for every color reproduction. Since the introduction of digital workflow (computer to film, to plate, and to press) in the industry, there are many experts who have come up with technologies and tools for color images that would benefit from improved color reproduction technologies. Screening is only an option of such technologies. In the digital printing environment, screening software can create the digital version of the amplitude modulate (AM) and also of the frequency modulated (FM) halftone screen. Screening software in the raster image processor (RIP) of the color managed workflow (CMW) at a digital printing press front-end platform (DFE) applies a digital dot pattern to the color image during printing.

Gray balance represents the combination of specific amounts of cyan, magenta, and yellow inks to produce a neutral shade of gray. With slight increases in cyan pigment required to produce a neutral gray, shifts in hue will occur with any imbalance of these three components. In addition to the color gamut, the gray balance is an additional requirement for pleasing color-reproduction. In large part, the imbalance is due to impurities of the inks, chromaticity deviation of the substrates, or other attributes. To establish the proper gray balance for a specific process, a full set of tint charts can be reproduced. Careful evaluation of the printed tint charts will provide the specific values for that specific reproduction process. The ISO 12647-7 document states that the gray balance can be printed and measured at the $\mathrm{CMY}$ overlap (overlap of $\mathrm{C}=50 \%, \mathrm{M}=40 \%$, and $Y=40 \%$ ). The deviation can be determined from the calculation of $\Delta H^{*}$ (deviation of hue, $h^{*}$ ) or $\Delta C^{*}$ (deviation of chroma, $\mathrm{c}^{*}$ ) and it requires the colorimetric data of CMY overlap printing from the $L^{*} a^{*} b^{*}$ model.
The quality of a color image reproduced through any printing process (digital or traditional) is largely influenced by the properties of paper. While paper is considered a commodity, its properties are a long way from being standardized (Wales, 2009). Additional attributes must be monitored in order to produce quality printed materials. The press operator must carefully manage several print parameters, such as the source colors (a source profile of ISO or ANSI standard), press calibration, press characterization (device destination profile), and the screening option. Without controlling these parameters to a print job a color mismatch would result.

\section{Amplitude Modulated (AM) Screening}

In AM screening, the dot frequency is constant and dot amplitude varies. AM screening breaks the image into dots of varying sizes, which are clustered together, at a fixed screen angle. For each color (CMYK) to be overprinted, the halftone CMYK separations need to be generated at a particular screen angle to avoid a moiré pattern. The dot is generated from a cluster of laser spots. A halftone cell (or grid) is then divided into a matrix of single recording dots (laser spots). Since there is always a physical and mathematical relationship among the number of gray levels, the resolution of the output device, and the screen ruling, there is always a tradeoff between resolution and the number of reproducible gray levels (Fleming, Dollak \& Fryzlewicz, 2004). The number of laser spots within a halftone cell depends on the following parameters: screen frequencies/screen angles, imagesetter resolution/ gray levels, dot size, and dot shape/dot patterns. The outcome of the clustering is a continuous tone pattern - lighter or darker shades or tones (See Figure 2).

\section{Frequency Modulated (FM) Screening}

In FM screening, the dot frequency varies, and dot size is constant ( $M a, 2003)$. FM screening, also referred to as stochastic screening, can be regarded as an electronic implementation of screenless printing (Chung \& Ma, 1995). The word stochastic means "involving a random variable" - it uses randomly placed dots instead of AM halftone dots aligned along the screen angle. FM screening uses a microdot placement (See Figure 2). The dot size is fixed, but the number of dots within a halftone cell varies according to the tonal value being imaged. The minimum size of the dots is limited only by the output device resolution and dots are measured in microns $(\mu \mathrm{m})$. A typical modern 2400 DPI output device has a laser spot size of 10.6 $\mu \mathrm{m}$ (microns, $10^{-6}$ ) and the size of a microdot is $21 \mu \mathrm{m}$ (Chung \& Ma, 1995). In FM screening, screen angles are not required when generating a CMYK separation. This results in the eradication of the moiré pattern in the printed image (Fleming, Dollak \& Fryzlewicz, 2004). FM screening, though, uses smaller dots that are not 
restricted to a fixed grid pattern. By varying the number of dots in a given area, any desired gray level can be generated. The dots are smaller than the AM screening, therefore FM screening can represent more detail and support higher resolution printing (See Figure 2).

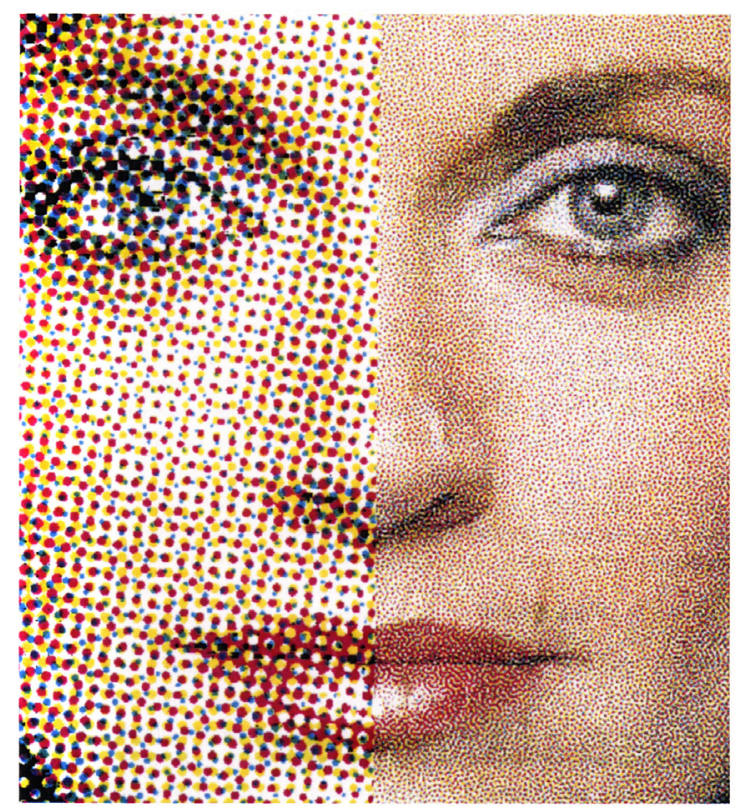

» Figure 2: AM (left) vs FM (right) Screened image (Source: Creo Review)

\section{Purpose of the research}

The purpose of this applied research was to demonstrate the use of a complete color managed workflow (CMW) and meet the specified $\mathrm{G} 7$ master compliance levels by creating and using output device ICC profiles. The experiment was conducted in a color managed digital color printing workflow (CMDCPW) to determine the effect that screening technologies have on the G7 master compliance: Comparison of Compliance with Amplitude Modulated (AM) vs. Frequency Modulated (FM) Screening of Multicolor Digital Printing. It was aimed at achieving the G7 master compliance through an ICC based CMW. As stated earlier, the $\mathrm{G} 7$ master compliance print evaluation can be achieved by use of the output device ICC profile for printing. This experiment adopts this method to achieve the compliance. G7 master compliance includes three compliance levels in the G7 master qualification: G7 Grayscale, G7 Targeted, and G7 Colorspace. These levels demonstrate $\mathrm{G} 7$ master capabilities of a print facility. The G7 calibration method, using the P2P251x target was NOT considered to derive the device NPDC to compare with G7 NPDC for print (or press) runs 1, 2, 3, etc.

\section{Limitations of the Research}

For this experiment, there were limitations to the technology used within the graphics program laboratory. Prior to printing and measuring the samples, the digital color output printing device and color measuring instruments (spectrophotometer and densitometer) were calibrated against the recommended reference. The print condition associated with this experiment was characterized by, but not restricted to, inherent limitations: colored images (TC1617x, ISO300, and ISO12647-7) chosen for printing, desired rendering intent applied, type of digital printer for proofing/printing, type of paper for printing, type of toner, resolution, screening technique, use of defined color output profiles, calibration data applied, and so on. Several variables affected the facsimile reproduction of color images in the CMDPW and most of them were mutually dependent. The scope of the research was limited to the color laser (electrophotographic) digital printing system (printing proof/printing) and other raw materials and the multiple types of color measuring devices and color management and control applications (data collection, data analysis, profile creation, and profile inspection) used at the university graphics laboratory. Findings were not expected to be generalizable to other CMDPW environments. It is quite likely, however, that others could find the method used and the data of this article meaningful and useful. The research methodology, experimental design, and statistical analysis were selected to align with the purpose of the research, taking into account the aforementioned limitations.

\section{Research methodology}

The digital color printing device used in this experiment is a Konica-Minolta bizHub C6000 Digital Color Press. It uses a Creo IC-307 raster image process (RIP) application (front-end system). A two-page custom test image $\left(12^{\prime \prime} \times 18^{\prime \prime}\right.$ size) was created for proofing and printing use for the experiment (See Figures 3 \& 3A). The test target contained the following elements: an ISO 300 and generic images for subjective evaluation of color, an ISO 12647-7 Control Strips (2013, three-tier), and a TC1617x target for gamut/profile creation. Colorimetric, Densitometric, and Spectrophotometric data were extracted by using an X-Rite Eye-One Spectrophotometer and an X-Rite i1iO Scanning Spectrophotometer from the color printed samples for the analysis. For both screening technologies ( $K=2)$, a total of 200 samples of target color images were printed, 100 prints for each screening method, noted by letter " $\mathrm{N}$ " $(\mathrm{N}=$ 100). Of 100 samples of each group, 80 samples ( $n$ $=80$ ) were randomly selected from each screening group, and measured, noted by the letter " $n$ " $(n=80)$. 
Aiming for G7 Master Compliance through a Color Managed Workflow:

Comparison of Compliance with Amplitude Modulated (AM) vs. Frequency Modulated (FM) Screening of Multicolor Digital Printing
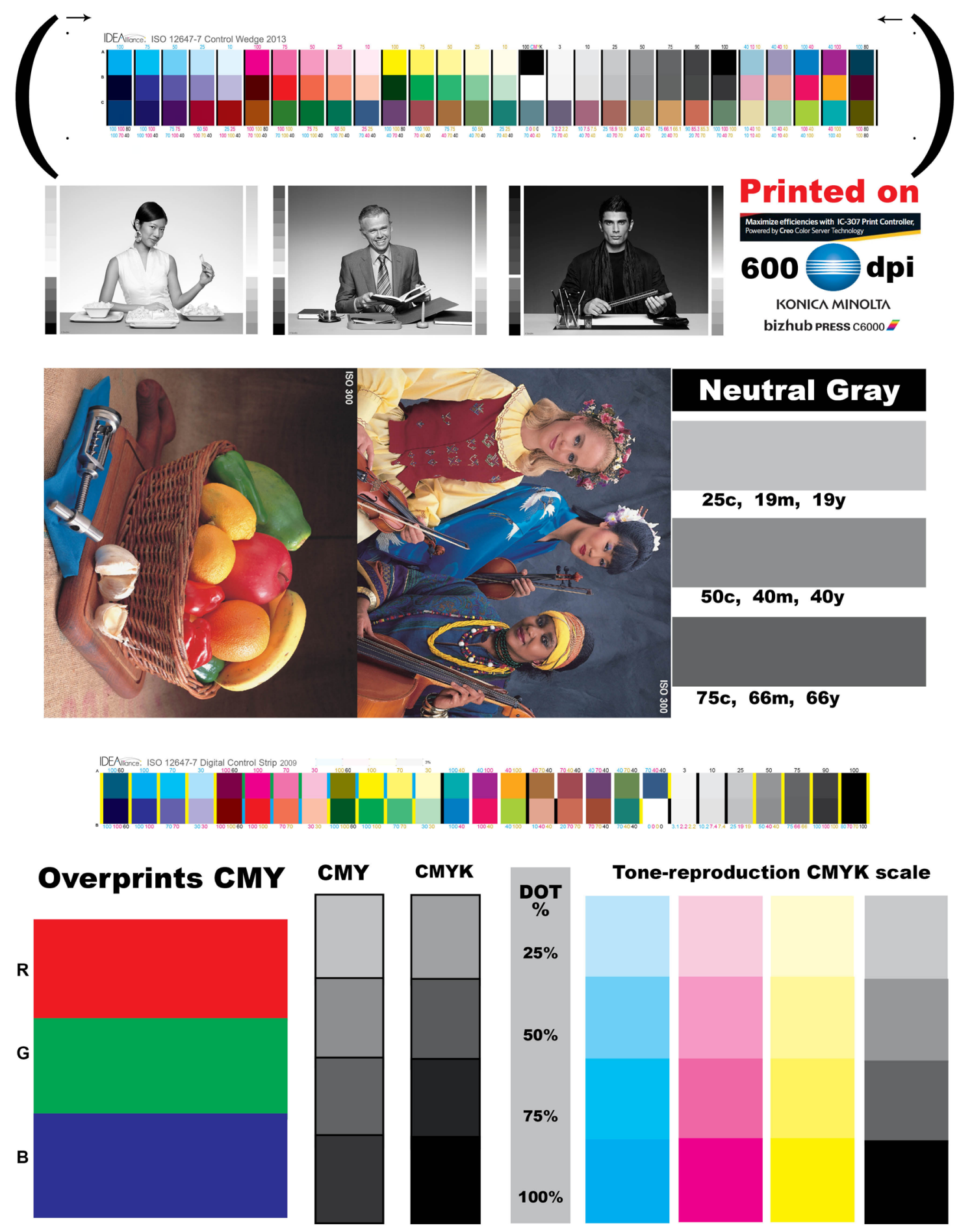

» Figure 3: Test Image for the experiment (PAGE 01) 


\section{Aiming for G7 Master Compliance through a Color Managed Workflow: \\ Comparison of Compliance with Amplitude Modulated (AM) vs. Frequency Modulated (FM) Screening of Multicolor Digital Printing}

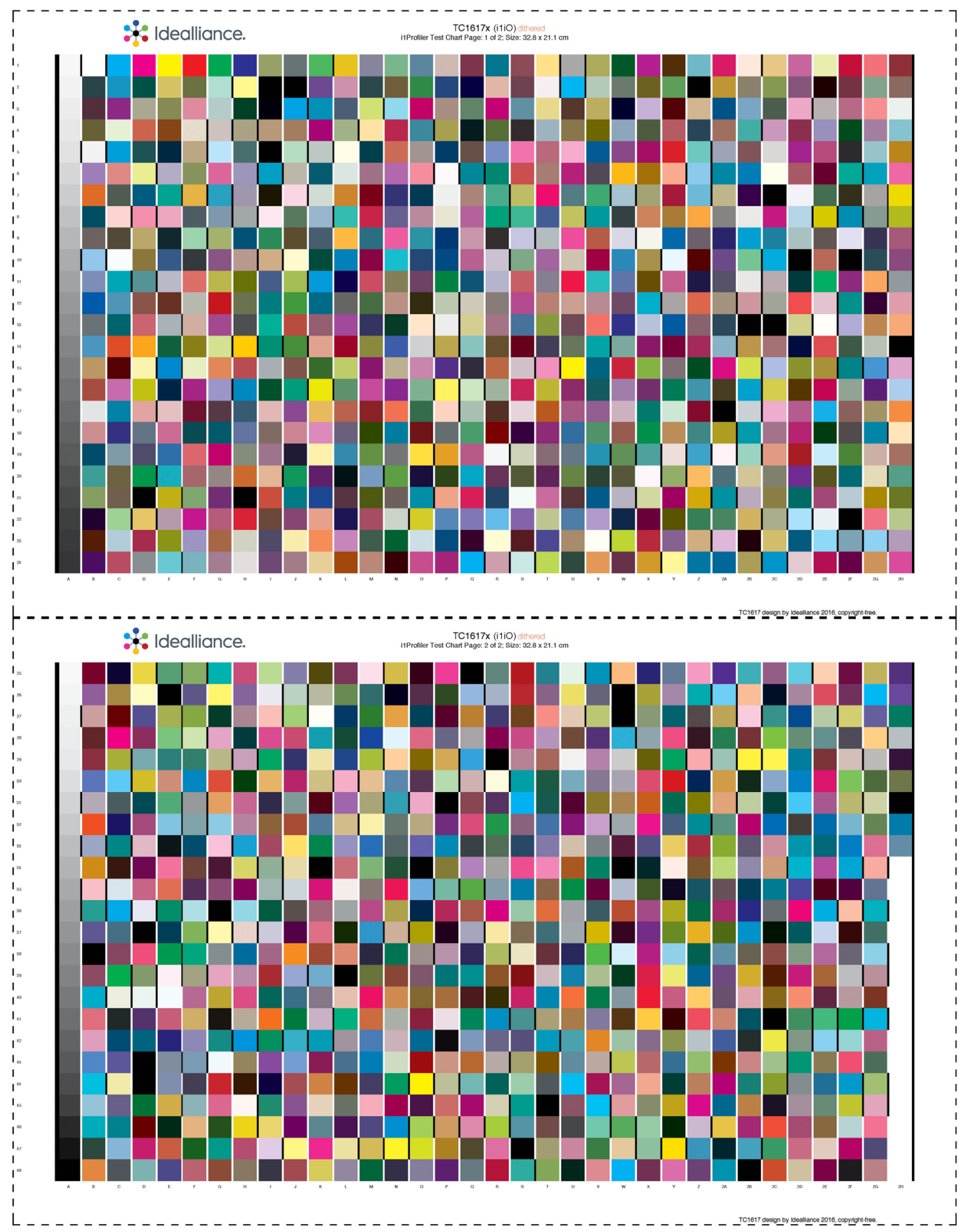

» Figure 3A: Test Image for the experiment (PAGE 02)

This sample size is needed to make the reliability of data is accurate. It is well documented that a large sample size is more representative of the sampling population (subjects). Each printed sheet is measured by using the scan- ning spectrophotometer, data was then saved, and later combined in the Chromix/IDEAlliance Curve 4 application for the analysis. Glass, G.V. \& Hopkins, K.D. (1996) provides an objective method to determine the sample size 
when the size of the total population is known. The following formula was used to determine the required sample size, which was 80 (n) printed sheets for this study:

$n=\left[\chi^{2} N P(1-P)\right] /\left[d^{2}(N-1)+\chi^{2} P(1-P)\right]$

$\mathrm{n}=$ the required sample size

$\chi^{2}=$ the table value of chi-square for 1 degree of freedom at the desired confidence level (3.84)

$\mathrm{N}=$ the total population size

$\mathrm{P}=$ the population proportion that it

is desired to estimate (.50)

$\mathrm{d}=$ the degree of accuracy express-

es as a proportion (.05)

\section{G7 Compliance for Digital Color Press (printer)}

Prior to printing the patches/target image, the printer was calibrated for both the screening options (AM and FM) according to its manufacturer specifications. A calibration process means standardizing the performance of the devices according to the device manufacturer specifications so that the results of the devices are repeatable. The calibration curve consists of the maximum printable densities of each color (CMYK) of both the screening techniques (See Figure 4). Test target TC1617x was used for the output device profile creation process.

\section{PRINTER CALIBRATION}

\section{AM Screening}
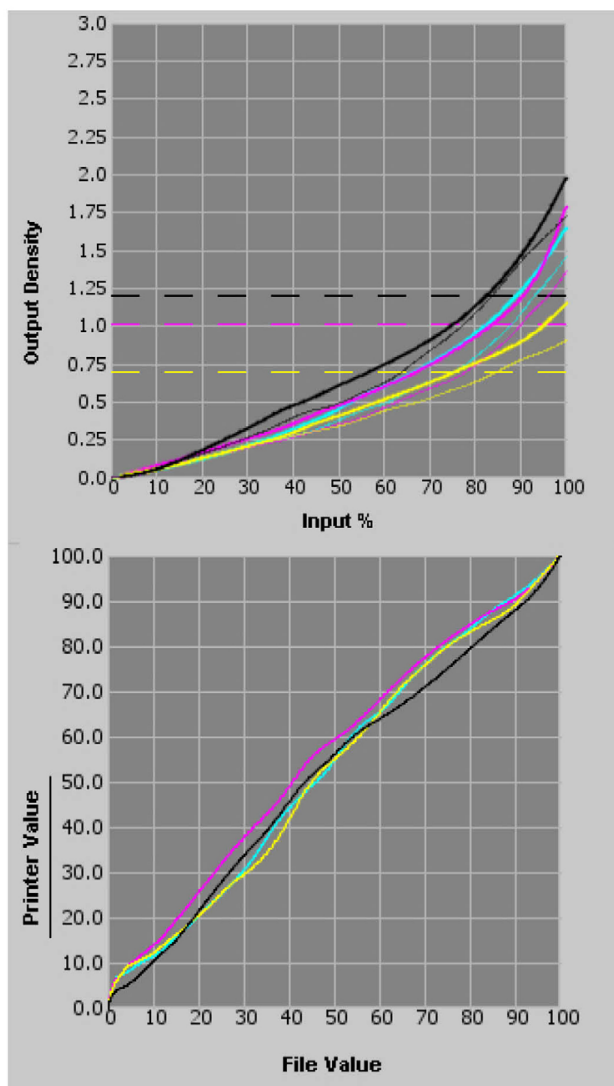

vs.
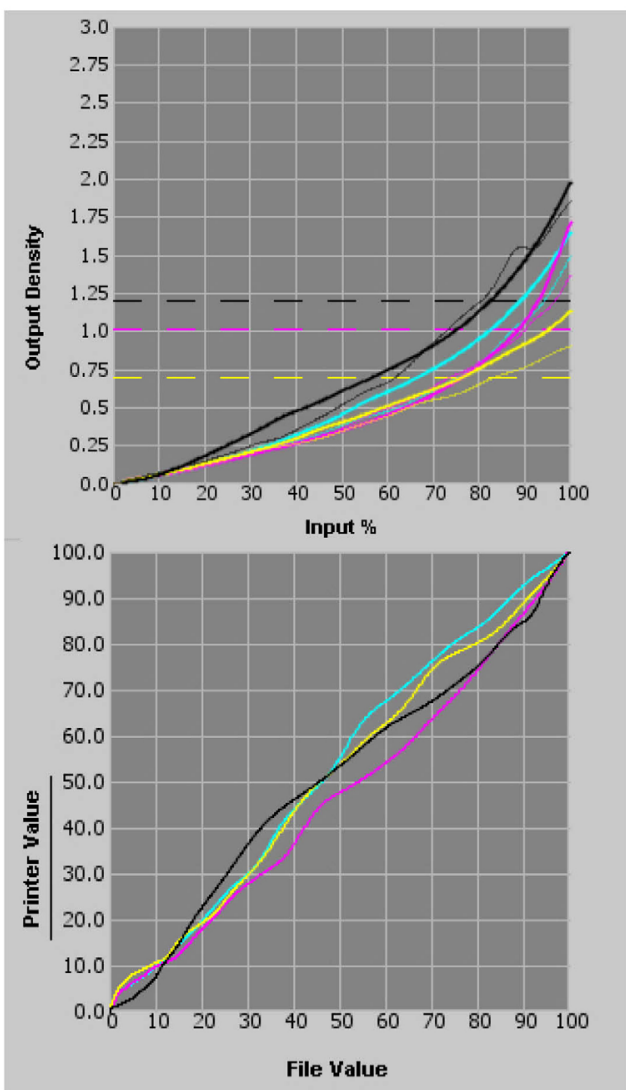

Measured Solid Ink Density (SID) of:

\begin{tabular}{|c|c|c|c|c|c|c|c|c|}
\hline \multicolumn{4}{|c|}{ AM Screening } & \multirow[b]{2}{*}{ vs. } & \multicolumn{4}{|c|}{ FM Screening } \\
\hline $\begin{array}{l}C \\
1.45\end{array}$ & $\begin{array}{c}M \\
1.36\end{array}$ & $\begin{array}{l}Y \\
0.90\end{array}$ & $\begin{array}{l}K \\
1.73\end{array}$ & & $\begin{array}{c}C \\
1.49\end{array}$ & $\begin{array}{l}M \\
1.37\end{array}$ & $\begin{array}{c}Y \\
0.90\end{array}$ & $\begin{array}{c}K \\
1.85\end{array}$ \\
\hline
\end{tabular}

vs.

FM Screening

» Figure 4: Calibration of AM vs. FM Screening 
In a generic color managed digital printing workflow, digital front-end (DFE) platforms (raster image processor or RIP) of digital printers (or presses) offer opportunities for the user (or press operator) to manipulate the output color quality to meet the expected demand of the customer. In order to print a quality halftone image, the user must carefully manage several print parameters, variables, and attributes which are associated with the digital printing process. For this experiment, printer resolution was reduced to 600 dots per inch (DPI) because one screening option (FM screen) in the front-end platform (RIP) was limited to only this setting. This is due to random micro-dot placement, without use of screen angles, for the dot reproduction of FM screening. However, the AM screening option offered the ability to set printer resolution at $600 \mathrm{DPI}, 1200 \mathrm{DPI}$, and $2400 \mathrm{DPI}$. In order to test the screening effect on the digital color output (DCO) only the 600 DPI resolution was selected for both screening technologies to keep the parameters/variables consistent throughout the experiment. Table 1 presents the variables, materials, conditions, and equipment associated with this experiment.

\section{Output Device Profiles (ODP) with AM and FM Screening for G7 Compliance}

The target image (TC1617x) was placed into an Adobe InDesign-CC layout of 12 " W $\times 18^{\prime \prime} \mathrm{H}$ size and a .PDF file was created without any image/color compression technique (see Figures 3 \& 3A). Hammermill brand, 100 LB matte-coated digital color printing paper 12 " $\times 18^{\prime \prime}$ was used for printing the samples in the experiment. Prior to printing the TC1617x target (See Figure 3A) for creating the device profile, the printer was calibrated. The calibration data (range of CMYK densities) were saved in the calibration lookup tables of the RIP and a calibration curve was created. A total of 100 sheets/ copies of TC1617x were printed with the calibration curve attached. Also, an amplitude modulated (AM) halftone screening technique with 190 lines per inch (LPI) and 600 DPI as the printer resolution was applied during the printing. No color management or color correction techniques were applied during the printing.

Printed patches of TC1617x were measured in CIE L* $a^{*} b^{*}$ space using the I1PROFILER application with an $X$-Rite spectrophotometer with an i1iO table and the data were run through this application. The printer profile was created and stored at the right location on the computer. The profile format version is 4.00 and it is considered as the Output Device Profile (ODP) of AM screening. This profile was used as a destination profile (DP) in the workflow. The source profile (SP) used in the experiment is a GRACoL2013 for characterized reference printing conditions-6 (CRPC-6). The same procedures as described above in this section were followed for creating the ODP for FM screening with an FM screened calibration curve and the $600 \mathrm{DPI}$ as the printer resolution.

\section{Table 1}

Experimental and Controlled Variables

\begin{tabular}{|c|c|}
\hline Variable & Material/Condition/Equipment \\
\hline Test image & Custom Test Target, 2 pages \\
\hline Control strips/targets & ISO 12647-7 (2013), TC1617x \\
\hline Other Images & $\mathrm{B} / \mathrm{W}$ and Color for Subjective Evaluation \\
\hline Profiling Software & X-Rite i1PROFILER 1.8 \\
\hline Profile Inspection Software & Chromix ColorThink-Pro 3.0 \\
\hline Image Editing Software & Adobe PhotoShop-CC \\
\hline Page Layout Software & Adobe InDesign-CC \\
\hline Source Profile (RGB) & Adobe 1998.icc \\
\hline Destination Profile (CMYK) & Custom, Konica-Minolta.icc \\
\hline Reference/Source Profile (CMYK) & GRACoL2013.icc \\
\hline Color Management Module (CMM) & Adobe (ACE) CMM \\
\hline Rendering Intents & Absolute \\
\hline Computer \& Monitor & Dell OPTIPLEX/LCD \\
\hline Raster Image Processor (RIP) & Creo IC-307 Print Controller \\
\hline Printer & Konica-Minolta bizHub C6000 Color Laser \\
\hline Achieved CMYK SID for all print runs (AM vs. FM) & $\mathrm{C}=1.47 ; \mathrm{M}=1.37 ; \mathrm{Y}=0.90 ;$ and $\mathrm{K}=1.79$ \\
\hline Screens and Screen Ruling & AM \& FM, 190 LPI for AM \\
\hline Print Resolution & $600 \times 600 \mathrm{DPI}$ \\
\hline Toner & Konica-Minolta Color Laser \\
\hline Type of Paper Weight/thickness & Hammermill 100LB Matte Coated, Sheetfed \\
\hline Type of Illumination/Viewing Condition & D50 \\
\hline Color Measurement Device(s) & $\begin{array}{l}\text { X-Rite Eye-One PRO Spectrophotometer with Status T, } \\
2^{\circ} \text { angle, and i1iO Scanning Spectrophotometer }\end{array}$ \\
\hline Data Collection/Analysis Software & Chromix/IDEAlliance Curve 4.0 \& MS-Excel \\
\hline
\end{tabular}


See Figure 5 for all output device profiles comparison (GRACoL 2013, AM vs FM Screened) with profile volume (gamut volume) and L* $\mathrm{a}^{*} \mathrm{~b}^{*}$ values of each profile used.

\section{Printing for G7 Compliance}

Each screening technique (AM or FM) used in the experiment (see Figure 6 ) was considered as a group, noted by letter " $K$ " $(K=2)$. A group involves a set of print parameters, such as: a digital halftone screening technique [amplitude modulate (AM) $\underline{\mathbf{O R}}$ frequency modulate

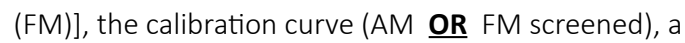
color source profile [General Requirements for Applications in Commercial offset Lithography for characterized reference printing conditions (GRACoL2013 for CRPC-6)], and a color destination profile of a digital press (AM

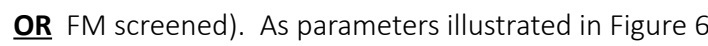
(Schematic Illustration of Sequence of Print Parameters for G7 Compliance), test target of $12^{\prime \prime} \times 18^{\prime \prime}$ was printed for use in the experiment. The test target contained the following elements: TC1617x target, ISO 12647-7 (2013) control strips, an ISO 300 and custom images of color and $b / w$ for subjective evaluation of color. A total of 100 sheets/samples were printed for each screening technique by enabling the color management technique at the RIP. The digital press AM or FM calibration curve, AM or FM screening destination profile, and the source profiles all were applied during the printing (see Figure 6).

\section{Press Run 1: Printing with ODP of AM Screening}

A total of 100 sheets/samples were printed (See Figure 6). The digital press calibration curve (AM Screened), AM screening destination profile, and the source profile (GRACoL 2013) all were applied during the printing. A total of 80 randomly pulled printed copies of TC1617x printed target images were measured against G7 ColorSpace GRACoL 2013 (CGATS21-2-CRPC6) in CIE L* a* b* space using an IDEAlliance (Chromix/Hutch Color) Curve 4.2.4 application interface with an X-Rite spectrophotometer with an i1iO table. The measured data were combined/averaged to run through this application (Curve 4.2.4). The combined data set was analyzed by using the Verify Tool of the application to determine the pass/ fail of G7 master compliance levels using G7 ColorSpace tolerances. Analyzed data from the experiment revealed that the printed colorimetric values (G7 Grayscale, G7 Targeted, and G7 Colorspace) are in match with the G7 master compliance levels (reference/target) colorimetric values (G7 Grayscale, G7 Targeted, and G7 Colorspace).

\section{Press Run 2: Printing with ODP of FM Screening}

A total of 100 sheets/samples were printed (See Figure 6). A total of 100 sheets/samples were printed by enabling the color management technique for the use of ODP of FM screening at the RIP. The digital press

\section{Output Device Profile Comparison}
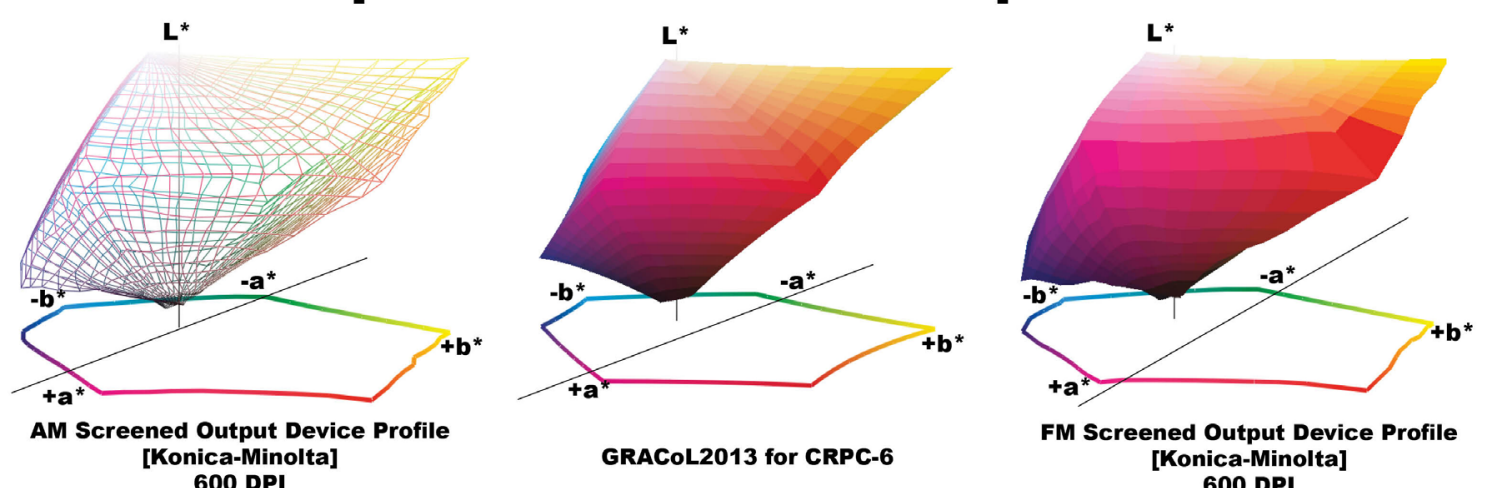

GRACoL2013 for CRPC-6

FM Screened Output Device Profile [Konica-Minolta] 600 DPI

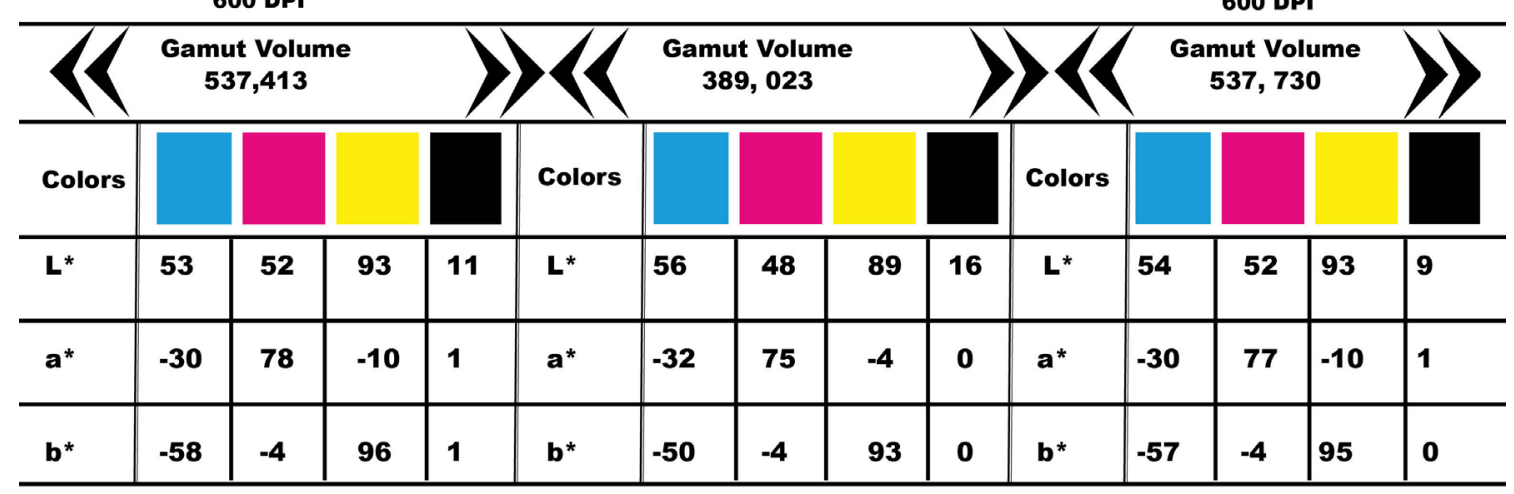

» Figure 5: Output Device Profiles Comparison 
AIMING FOR G7 MASTER COMPLIANCE THROUGH A COLOR MANAGED WORKFLOW: SEQUENCE OF PRINT PARAMETERS FOR THE COMPLIANCE

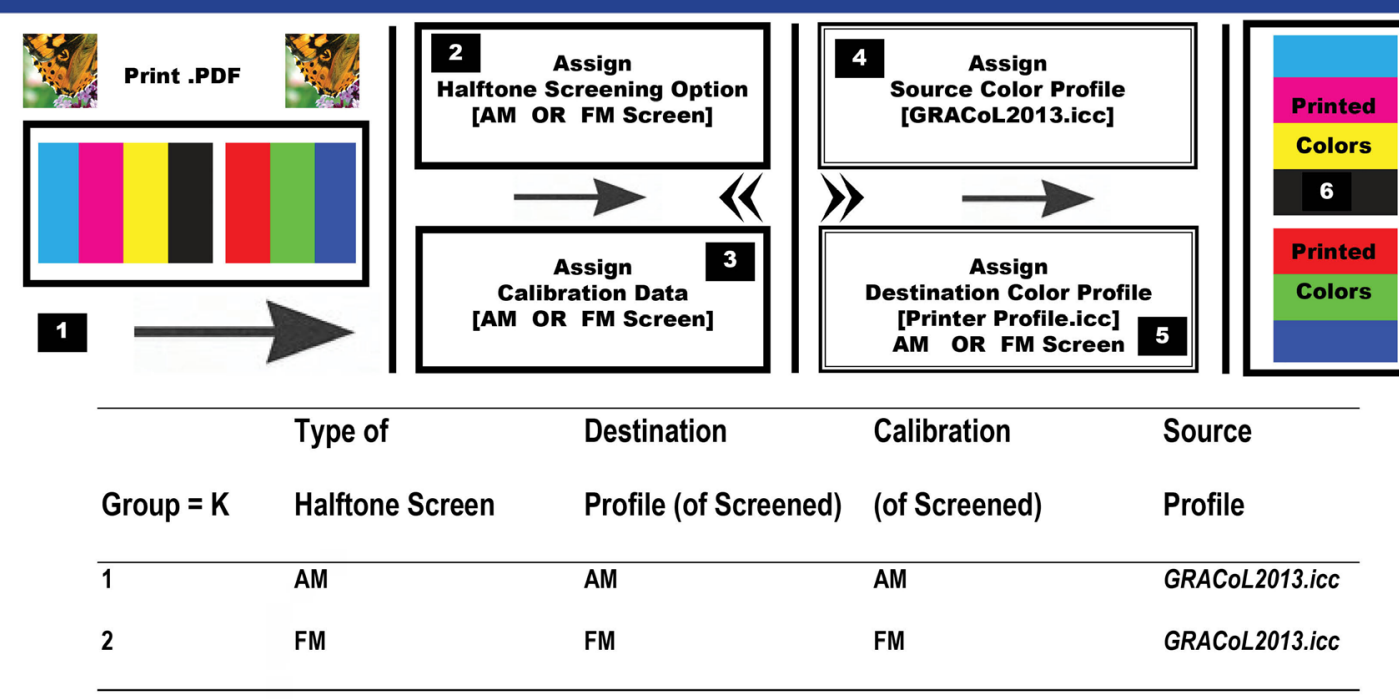

» Figure 6: Schematic Illustration of Sequence of Print Parameters for G7 Compliance

FM screened calibration curve and the resolution both were applied during the printing. A total of 80 randomly pulled printed copies of TC1617x printed target images were measured against G7 ColorSpace GRACoL 2013 (CGATS21-2-CRPC6) in CIE L* a* b* space using an IDEAlliance (Chromix/Hutch Color) Curve 4.2.4 application interface with an X-Rite spectrophotometer with an i1iO table. The measured data were combined/averaged to run through this application. Color measurement and analysis steps used in the printing with ODP process AM screening (previous section) were applied/ followed for printing with the FM screening printing process. Printed colors with ODP of FM screening were also found to be very accurate and efficient.

\section{Data analysis \& research findings}

The colorimetric computation methods for G7 compliance were used to analyze the collected data and presented in the following pages/tables. Subjective judgment on color difference or any deviation was not used in this study. The subjective judgment of color difference could differ from person to person. For example, people see colors in an image not by isolating one or two colors at a time (Goodhard \& Wilhelm, 2003), but by mentally processing contextual relationships between colors where the changes in lightness (value), hue, and chroma (saturation) contribute independently to the visual detection of spatial patterns in the image (Goodhard \& Wilhelm, 2003). Instruments, such as colorimeters and spectrophotometers, could eliminate the subjective errors of color evaluation perceived by human beings. In comparing the color differences between two colors, a higher deviation ( $\Delta \mathrm{E}$ or $\Delta \mathrm{H}$ or the $\Delta \mathrm{C}$ ) is an indication that there is more color difference and a lesser deviation ( $\Delta \mathrm{E}$ or $\Delta \mathrm{H}$ or the $\Delta \mathrm{C}$ ) is an indication of less color difference.

The data for each screening group were generated from the printed TC1617x target image (see Figure 3A) by using an Eye-One-Pro spectrophotometer with an interface application, such as the IDEAlliance Curve 4.2.4 against the GRACoL2013_CRPC6 reference data. The test chart (TC) $1617 x$ is a new CMYK printer characterization target combining the unique patch values in the standard IT8.7/4 target with all the patch values in columns 4 and 5 of the P2P51 target. The letter " $x$ " distinguishes the final version from earlier prototype versions circulated during development. The TC1617x maintains the same patch count as the IT8.7/4 (1,617 - hence the name is TC1617x) by removing 29 duplicate patches from the IT8.7/4 and replacing them with the 29 patches in columns 4 and 5 of the P2P51 that were absent in the IT8.7/4. Data derived from the TC1617x target image is the difference between the characterization data set (TC1617x) and the printed sample. The reference file content for this image (TC1617x) is the CMYK dot percentage values and nominal CIE L* a* b* characterization data values for the GRACoL2013-CRPC6 reference. Analyzed G7 master compliance levels (reference/target) data (G7 Grayscale, G7 Targeted, and G7 ColorSpace) with G7 colorimetric formulae and formats were presented in the following sections for each of the levels. 


\section{CIE L*a* b*, Delta L* Delta $E$ and Delta Chroma $(\Delta \mathrm{L}, \Delta \mathrm{E}$ and $\Delta \mathrm{C})$}

Colorimetric values of printed colors against original colors and the deviations (Deltas) can be used to determine the visual variation in overall colors, hue, chroma, and lightness. The $a^{*}, b^{*}$ coordinates correspond approximately to the dimensions of redness - greenness and yellowness - blueness respectively in the CIE L* a* b* color space and are orthogonal to the $L^{*}$ dimension. Hence a color value whose coordinates $a^{*}=b^{*}=0$ is considered achromatic regardless of its $L^{*}$ value. Calculation of $\Delta H^{*}$ requires colorimetric data from the $L^{*} a^{*} b^{*}$ model.

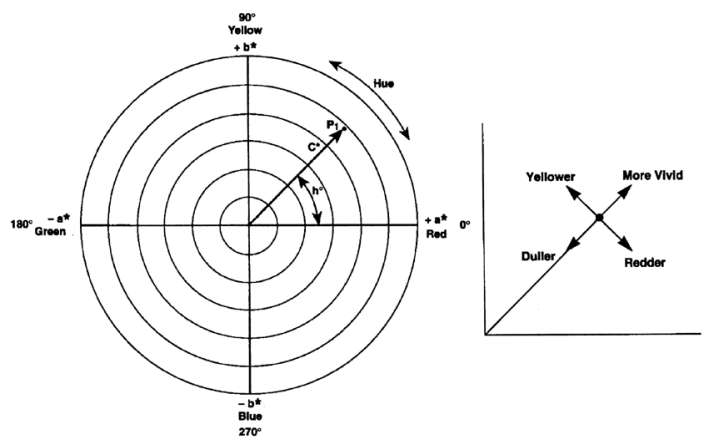

» Figure 7: Schematic of $L^{*} a^{*} b^{*} \& c^{*}, h^{*}$ Coordinates

Metric hue angle $\mathrm{h}^{*}$ and $\mathrm{C}^{*}$ are defined by the following formulas (Morovic, Green, \& MacDonald, 2002).

$h *_{a b}=\tan ^{-1}\left(\frac{b *}{a *}\right)$

Where: $a^{*}, b^{*}$ are chromaticity coordinates in $L^{*} a^{*} b^{*}$ color space

Chroma $\left(\mathrm{C}^{*}\right)=\left[\mathrm{a}^{2}+\mathrm{b}^{2}\right]^{1 / 2}$

Where: $a^{*}, b^{*}$ are chromaticity coordinates in $L^{*} a^{*} b^{*}$ color space

Calculation of $\Delta C^{*}$ (of two colors) and $\Delta L^{*}$ requires colorimetric data from the $L^{*} a^{*} b^{*}$ model. Difference in the chroma $C^{*}$ of two colors (Reference vs. Printed) can be calculated by using the following formula (Green et al., 2002).

$\Delta$ Chroma $(\Delta \mathrm{C})=\mathrm{C}{ }_{1}-\mathrm{C} *_{2}$

Where: $1=\mathrm{C}^{*}$ of Reference Color and $2=\mathrm{C}^{*}$ of Printed Color

Assessment of color is more than a numeric expression. It is an assessment of the difference in the color sensation (delta) from a known standard. In the CIELAB color model, two colors can be compared and differentiated. The expression for these color differences is expressed as $\Delta E$ (Delta $E$ or Difference in Color Sensation). The fol- lowing equation is used to calculate the $\triangle E$ (Committee for Graphic Arts Technologies Standards - CGATS, 2003)

$$
\Delta E^{*}=\sqrt{\left(L_{1}-L_{2}\right)^{2}+\left(a_{1}-a_{2}\right)^{2}+\left(b_{1}-b_{2}\right)^{2}}
$$

Where: $1=$ Reference Color and $2=$ Printed Color

Chromaticness difference $(\Delta C h)$ is the difference between the reference chroma $\left(a{ }_{1}\right.$ and $\left.b^{*}{ }_{1}\right)$ and the measured chroma $\left(a^{*}{ }_{2}\right.$ and $\left.b^{*}{ }_{2}\right)$ of a gray balance control patch (C50, M40, Y40). Weighted Delta Chroma $(\mathrm{w} \Delta \mathrm{Ch})$ is the delta $\mathrm{Ch}$ value after it is passed through a weighting curve that reduces the significance of Ch errors in the darker regions of the color. The weighting function is defined in the G7 specifications ([Technical Report (TR) 015] and the G7 master pass/ fail document as follows (Chromix, Inc., 2019):

$\mathrm{w} \Delta \mathrm{Ch}=\Delta \mathrm{Ch} \times[1-\max (0,(\%-50) / 50 \times 0.75)]$

Delta $L^{*}\left(\Delta L^{*}\right)$ is the difference in the lightness between the reference and measured sample lightness regardless of any color. This makes $\Delta L^{*}$ the perfect metric for measuring tonality [Neutral Print Density Curve (NPDC)] error in G7. Colorimetrically, $\Delta L^{*}$ is the result of subtracting the $L^{*}$ of measured sample value from the reference $L^{*}$, as follows:

$\Delta \mathrm{L} *=\mathrm{L}^{*}-\mathrm{L}_{2}$

Where: $1=L^{*}$ of Reference Color and $2=L^{*}$ of Printed Color

Weighted Delta $L^{*}\left(w \Delta L^{*}\right)$ is the delta $L^{*}$ value after it is passed through a weighting curve that reduces the significance of $L^{*}$ errors in the darker regions of the color. The weighting function is identical to that for $w \Delta C h$, as follows (Chromix, Inc., 2019):

$\mathrm{w} \Delta \mathrm{L}^{*}=\Delta \mathrm{L}^{*} \times[1-\max (0,(\%-50) / 50 \times 0.75)]$

\section{Overall Color Variation $(\Delta E)$ of AM Screened (TC1617x image) vs. GRACoL 2013 Ref.}

The CIE L* $a * b *$ values associated with the CMYK+RGB colors AM screened image vs. G7 ColorSpace-GRACoL 2013 [CGATS21-2-CRPC6 (reference)] are compiled in Table 2. Numerical color differences $(\triangle E)$ were found when comparing the colors of an AM screened printed image vs. G7 ColorSpace at all seven colors (CMYK+RGB). Also, noticeable visual color differences were found in the solid color area [lightness, color hue and chroma]. Overall, both groups of images have similar colors (See Figure 8) with the exception of the printed image consisting of higher $\mathrm{L}^{*}$ for red, magenta, and green, etc. This results in producing the higher $\Delta \mathrm{E}$ for these colors. 
Table 2

Overall Color Variation of CMYK+RGB: AM Screened Image (TC1617x) vs. G7 ColorSpace

\begin{tabular}{|c|c|c|c|c|c|c|c|}
\hline \multirow{3}{*}{ Color(s) } & \multicolumn{3}{|c|}{ AM Screened Image } & \multicolumn{3}{|c|}{ G7 ColorSpace / Target } & \multirow{3}{*}{$\begin{array}{c}\text { Color } \\
\text { Difference } \\
\Delta \mathrm{E}\end{array}$} \\
\hline & $L^{*}$ & $a^{*}$ & $b^{*}$ & $L^{*}$ & $a^{*}$ & $b^{*}$ & \\
\hline & \multicolumn{3}{|c|}{$\begin{array}{l}\text { Color } 1 \\
\mathbf{N}=\mathbf{8 0}\end{array}$} & \multicolumn{3}{|c|}{$\begin{array}{l}\text { Color } 2 \\
\mathbf{N}=\mathbf{N} / \mathbf{A}\end{array}$} & \\
\hline White (W) & 97.22 & 2.79 & -9.47 & 97.22 & 2.79 & -9.47 & 0.00 \\
\hline Cyan & 57.44 & -31.3 & -54.11 & 57.39 & -36.88 & -55.85 & 1.99 \\
\hline Magenta & 51.49 & 76.06 & -5.38 & 49.21 & 77.92 & -7.10 & 2.40 \\
\hline Yellow & 90.98 & -5.36 & 91.29 & 91.09 & -2.44 & 92.91 & 1.62 \\
\hline Black (K) & 13.46 & 0.34 & -0.11 & 16.30 & 0.24 & -0.74 & 1.97 \\
\hline Red & 50.64 & 67.72 & 47.65 & 48.19 & 70.73 & 48.27 & 2.59 \\
\hline Green & 53.39 & -66.80 & 26.68 & 51.25 & -66.87 & 24.48 & 2.29 \\
\hline Blue & 26.79 & 20.05 & -51.57 & 25.62 & 21.18 & -50.24 & 1.60 \\
\hline TAC 300 & 24.33 & -0.07 & -1.73 & 23.56 & 0.45 & -1.35 & 1.02 \\
\hline TAC 400 & 9.74 & 0.55 & -0.97 & 8.99 & 0.17 & 0.61 & 1.72 \\
\hline
\end{tabular}

This higher color deviation (red, magenta, and green) could be the result of the substrate (paper) and inks used (age, condition, quality, etc.). These are the darker colors which produced lower $\mathrm{L}^{*}$ value and, in turn, affected the higher deviation. The $2 \mathrm{D}$ color gamut comparison (see Figure 8 ) reveals that the colors of the printed image closely match the reference colors. The goal was to determine the deviations among various attributes of color between these two groups of colors. The comparison is an indication that, in a color managed workflow (CMW), color matching of a target image can be achieved from device to device regardless of device color characterization and original colors. Subjective judgment was not used for the color comparison.

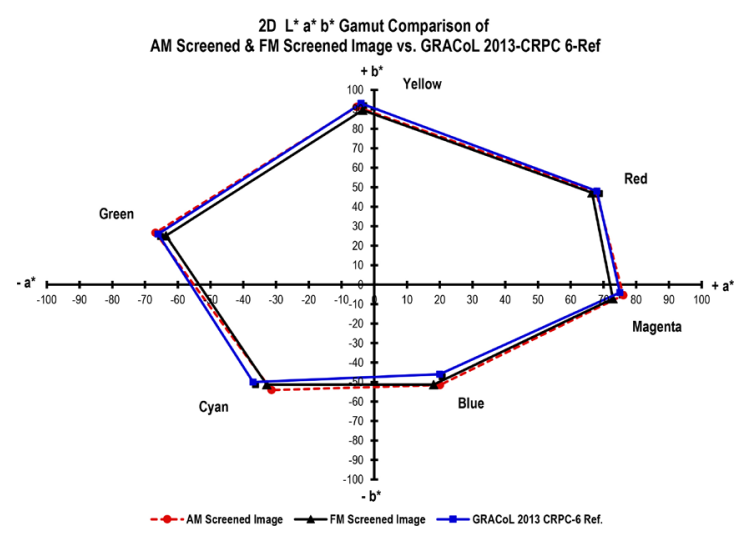

» Figure 8: AM Screened and FM Screened Image vs. GRACoL 2013-CRPC-6 Ref.

\section{Overall Color Variation $(\Delta E)$ of FM Screened (TC1617x image) vs. GRACoL 2013 Ref.}

The CIE L* a* $b *$ values associated with the CMYK+RGB colors FM screened image vs. G7 ColorSpace-GRACoL 2013 [CGATS21-2-CRPC6 (reference)] are compiled in
Table 3. Numerical color differences $(\Delta E)$ were found when comparing (See Figures 8) the colors of the FM screened printed image vs. G7 ColorSpace at all seven colors (CMYK+RGB). Also, noticeable visual color differences were found in the solid color area [lightness, color hue and chroma]. Overall, both groups of images have similar colors with the exception of the printed image consisting of higher $L^{*}$ for red, green, and blue, etc. This results in producing the higher $\Delta \mathrm{E}$ for these colors. See Figure 9 (scanned printed images) for visual comparison of the printed colors of the screening method applied.
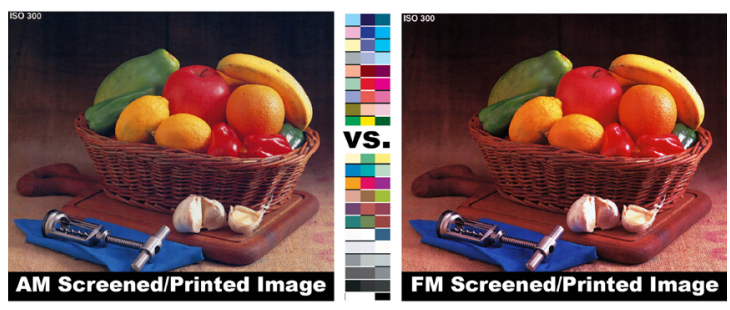

» Figure 9: AM Screened Printed vs. FM Screened Printed Images (colors)

This higher color deviation (red, green, and blue) could be the result of the screening technique applied. These are the darker colors which produced lower $L^{*}$ value and, in turn, affected the higher deviation. The 2D color gamut comparison (see Figure 8) reveals that the colors of the printed image closely match the reference colors. The goal was to determine the deviations among various attributes of color between these two groups of colors. The comparison is an indication that, in a color managed workflow (CMW), G7 master compliance for color matching of a target image can be achieved. Subjective judgment was not used for the color comparison.

In addition to the colorimetric comparison of individual colors (Tables 3 and 4) of both screening groups with G7 ColorSpace, the $\mathrm{G} 7$ master compliance colorimetric deviation ( $w \Delta \mathrm{Ch}$ and 
Table 3

Overall Color Variation of CMYK+RGB: FM Screened Image (TC1617x) vs. G7 ColorSpace

\begin{tabular}{|c|c|c|c|c|c|c|c|}
\hline \multirow{3}{*}{ Color(s) } & \multicolumn{3}{|c|}{ FM Screened Image } & \multicolumn{3}{|c|}{ G7 ColorSpace / Target } & \multirow{3}{*}{$\begin{array}{c}\text { Color } \\
\text { Difference } \\
\qquad \mathrm{E}\end{array}$} \\
\hline & $L^{*}$ & $a^{*}$ & $b^{*}$ & $L^{*}$ & $a^{*}$ & $b^{*}$ & \\
\hline & \multicolumn{3}{|c|}{$\begin{array}{l}\text { Color } 1 \\
\mathbf{N}=\mathbf{8 0}\end{array}$} & \multicolumn{3}{|c|}{$\begin{array}{c}\text { Color } 2 \\
\mathbf{N}=\mathbf{N} / \mathbf{A}\end{array}$} & \\
\hline White (W) & 97.03 & 2.87 & -9.98 & 97.03 & 2.87 & -9.98 & 0.00 \\
\hline Cyan & 56.34 & -32.82 & -51.34 & 57.27 & -36.77 & -56.22 & 1.87 \\
\hline Magenta & 50.06 & 72.88 & -7.15 & 49.11 & 77.85 & -7.38 & 1.48 \\
\hline Yellow & 89.73 & -3.57 & 89.57 & 90.92 & -2.35 & 92.53 & 1.17 \\
\hline Black (K) & 15.73 & 0.05 & 0.47 & 16.28 & 0.26 & -0.81 & 1.35 \\
\hline Red & 50.65 & 66.50 & 47.19 & 48.09 & 70.67 & 48.07 & 2.82 \\
\hline Green & 53.96 & -63.60 & 25.14 & 51.15 & -66.70 & 24.22 & 2.95 \\
\hline Blue & 27.49 & 18.13 & -51.30 & 25.57 & 21.16 & -50.44 & 2.76 \\
\hline TAC 300 & 24.30 & -0.28 & 0.53 & 23.51 & 0.47 & -1.47 & 2.31 \\
\hline TAC 400 & 10.63 & 0.44 & 0.21 & 8.99 & 0.16 & 0.62 & 1.17 \\
\hline
\end{tabular}

Table 4

G7 Master Compliance Levels G7 Grayscale of AM Screen, FM Screen vs. G7

\begin{tabular}{|c|c|c|c|c|}
\hline \multirow{2}{*}{ All Metrics } & \multirow{2}{*}{$\frac{\text { Black (K) }}{\mathrm{w} \Delta \mathrm{L}^{*}}$} & \multicolumn{2}{|c|}{ CMY (Overlap) } & \multirow{2}{*}{ G7 Tolerance } \\
\hline & & $w \Delta \mathrm{L}^{*}$ & w $\Delta C h$ & \\
\hline \multicolumn{5}{|c|}{ G7 Grayscale of AM Screened Image (Tonality/Gray Balance) } \\
\hline Average & 0.90 & 0.39 & 0.89 & 1.50 \\
\hline Maximum & 2.37 & 1.18 & 1.72 & 3.00 \\
\hline \multicolumn{5}{|c|}{ G7 Grayscale of FM Screened Image (Tonality/Gray Balance) } \\
\hline Average & 0.91 & 0.89 & 1.12 & 1.50 \\
\hline Maximum & 2.16 & 1.92 & 2.4 & 3.00 \\
\hline
\end{tabular}

\section{Table 4A:}

G7 Master Compliance Levels G7 Targeted of AM Screen, FM Screen vs. G7

\begin{tabular}{|c|c|c|c|c|}
\hline All Metrics & $\Delta E 2000$ & G7 Tolerance & Maximum & G7 Tolerance \\
\hline \multicolumn{5}{|c|}{ G7 Targeted of AM Screened Image } \\
\hline Substrate & 0.00 & 3.00 & & \\
\hline $\mathbf{K}$ & 1.97 & 5.00 & & \\
\hline CMY & & & 2.40 & 3.5 \\
\hline RGB & & & 2.59 & 4.3 \\
\hline \multicolumn{5}{|c|}{ G7 Targeted of FM Screened Image } \\
\hline Substrate & 0.00 & 3.00 & & \\
\hline $\mathbf{K}$ & 1.35 & 5.00 & & \\
\hline CMY & & & 1.87 & 3.5 \\
\hline RGB & & & 2.95 & 4.3 \\
\hline
\end{tabular}

$\mathrm{w} \Delta \mathrm{L}$ ) values for all the three levels (G7 Grayscale, G7 Targeted and $\mathrm{G} 7$ Colorspace) are in close match with the established tolerances for the G7 (see Table 4, 4A, and 4B) including the Neutral Print Density Curve [NPDC (CMY)] and NPDC (K).

\section{Summary/Conclusions}

This experiment used an output device ICC profile to achieve the compliance. G7 master compliance includes three compliance levels in the $\mathrm{G} 7$ master qualification: G7 Grayscale, G7 Targeted, and G7 Colorspace. These levels demonstrate G7 master capabilities of
Table 4B:

G7 Master Compliance Levels G7 Colorspace of AM Screen, FM Screen vs. G7

\begin{tabular}{|c|c|c|}
\hline All Metrics & $\Delta \mathrm{E} 2000$ & G7 Tolerance \\
\hline \multicolumn{3}{|c|}{ G7 Colorspace of AM Screened Image } \\
\hline Average & 1.28 & 3.5 \\
\hline $95 \%$ & 2.33 & 5.0 \\
\hline \multicolumn{3}{|c|}{ G7 Colorspace of FM Screened Image } \\
\hline Average & 1.71 & 3.5 \\
\hline $95 \%$ & 3.53 & 5.0 \\
\hline
\end{tabular}

a print facility. The experiment was conducted in a Color Managed Digital Printing Workflow (CMDPW). It was aimed at achieving the G7 master compliance through an ICC based color managed workflow (CMW). The G7 calibration method, using the P2P251x target, was NOT used to derive the device NPDC to compare with G7 NPDC for print (or press) runs 1, 2, 3, etc.

The conclusions of this study are based upon an analysis of colorimetric data, visual assessment, and associated findings. The experiment analyzed the comparison of G7 compliance of two screening technologies. The guiding objectives of this study allowed testing of an 
accepted color management practice to gain a better understanding of the presumptions associated with the application of an output device profile (ODP). The experiment examined the importance of calibration, characterization and the color evaluation processes of the digital press which was capable of printing colors to match or be in proximity of G7 master compliance levels.

It is evident that integration of device profiles is important in a CMW and it also enables/allows the workflow process to meet the G7 compliance levels via an ICC based CMW, instead of using G7 calibration methodology. Selection of screening method is based on individual preference because both screening options proved to be within the $\mathrm{G} 7$ compliance. This study represented specific printing or testing conditions. The images, printer, instrument, software, and paper that were utilized are important factors to consider when evaluating the results. The findings of the study cannot be generalized to other digital printing workflow. However, other graphic arts educators, industry professionals, and researchers may find this study meaningful and useful.

\section{References}

Chromix, Inc. (2019) Curve 4.2.4 Software Manual. Seattle, Chromix, Inc.

Chung, R. \& Ma, L. L. (1995) Press Performance Comparison between AM and FM Screening. TAGA Proceedings 1995, Rochester, New York, TAGA.

Committee for Graphic Arts Technologies StandardsCGATS (2003) Graphic technology - spectral measurement and colorimetric computation for graphic arts image. (ANSI/CGATS.5-2003). Reston, The Association for Suppliers of Printing and Publishing Technologies, p.29.

Fleming, P., Dollak, J. \& Fryzlewicz, S. (2008) Stochastic Screening: What to do When Your Rip Doesn't Support It and Comparison with Conventional Screening on an Offset Press. Journal of Graphic Technology. 1 (3), 15-23

Glass, G. V. \& Hopkins, K. D. (1996) Statistical Methods in Education \& Psychology. Boston, Allyn \& Bacon.

Goodhard, M. M. \& Wilhelm, H. (2003) A new test method based on CIELAB colorimetry for evaluating the permanence of pictorial images. Wilhelm Imaging Research, Inc. Available from: http://www.wilhelm-research.com [Accessed: 20th August 2019].

International Digital Enterprise Alliance- IDEAlliance (2014) IDEAlliance Guide to Print Production. Alexandria, IDEAlliance.

Ma, L.L. (2003) Understanding Digital Halftones. Journal of Visual Communications. 130-136.

Morovic, J. (2002) Colour gamut mapping. In: Green, P. \& MacDonald, L. (eds.) Colour Engineering Achieving Device Independent Colour. New York, John Wiley \& Sons, pp. 297-314
Novaković, D. \& Avramović, D. (2012) Influence of Printing Surface Attributes on Print Quality in Electrophotography. Technical Gazette. 19 (2), 295-301.

Pnueli, Y. \& Bruckstein, A. (1996) Gridless Halftoning: A Reincarnation of the Old Method. Graphical Models and Image Processing. 58 (1), 38-64. Available from: doi: 10.1006/gmip.1996.0003

Wales, T (2009) Paper: The Fifth Color. In: IPA Bulletin. Alexandria, IDEAlliance. 


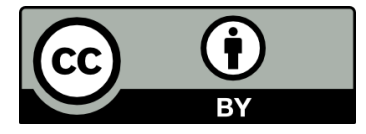

(C) 2021 Authors. Published by the University of Novi Sad, Faculty of Technical Sciences, Department of Graphic Engineering and Design. This article is an open access article distributed under the terms and conditions of the Creative Commons Attribution license 3.0 Serbia (http://creativecommons.org/licenses/by/3.0/rs/). 